Review

\title{
Salamander Regeneration as a Model for Developing Novel Regenerative and Anticancer Therapies
}

\author{
Jonathan Fior ${ }^{凶}$ \\ Innovative Bioresearch, Milan, Italy. \\ $\triangle$ Corresponding author: Jonathan Fior. E-mail: jonathan.fior82@gmail.com. \\ (c) Ivyspring International Publisher. This is an open-access article distributed under the terms of the Creative Commons License (http://creativecommons.org/ \\ licenses/by-nc-nd/3.0/). Reproduction is permitted for personal, noncommercial use, provided that the article is in whole, unmodified, and properly cited.
}

Received: 2014.06.23; Accepted: 2014.09.04; Published: 2014.09.20

\begin{abstract}
Among vertebrates, urodele amphibians are the only tetrapods with the ability to regenerate complex structures such as limbs, tail, and spinal cord throughout their lives. Furthermore, the salamander regeneration process has been shown to reverse tumorigenicity. Fibroblasts are essential for salamander regeneration, but the mechanisms underlying their role in the formation of a regeneration blastema remain unclear. Here, I review the role of fibroblasts in salamander limb regeneration and how their activity compares with that of human fibroblasts. In addition, the question of whether salamander blastema tissue could induce regeneration and tumor regression in animals with a limited regeneration ability is discussed. A deeper understanding of these processes may lead to the development of novel regenerative and anticancer therapies.
\end{abstract}

Key words: salamander regeneration; wound healing; tumor progression; fibroblasts.

\section{Introduction}

Fibroblasts are prototypical mesenchymal cells responsible for synthesizing the extracellular matrix, thus preserving the structural integrity of connective tissues [1]. In addition, fibroblasts play a crucial role in wound healing and tumor development [1]; therefore, these cells are of great interest for the study of these processes. Among vertebrates, urodele amphibians are the only tetrapods with the ability to regenerate complex structures such as limbs, tail, and spinal cord throughout their lives [2]. However, this remarkable turnover does not result in a high incidence of tumor formation [3], suggesting that a tight control system is in place that prevents uncontrolled cell proliferation and therefore cancer.

A possible hypothesis for the origin of human cancer is that during the course of evolution, humans lost an advanced regeneration ability as well as the associated control system, resulting in a more permissive environment for cancer development. Interestingly, humans seem to retain a silent regeneration potential in the form of quiescent stem cells. For ex- ample, humans cannot regenerate cardiac tissue after myocardial infarction, despite the presence of adult cardiac stem cells [4]. One of the most striking differences between human and salamander healing is the nature of fibroblast activity after tissue injury. In contrast with human fibroblasts, salamander fibroblasts initiate a dedifferentiation program following injury. Fibroblast dedifferentiation is a crucial step for the formation of a regeneration blastema, a mass of undifferentiated proliferating cells responsible for the regeneration of complex structures such as limbs. Although other cell types contribute to blastema formation, fibroblasts appear to play a central role $[5,6]$. Here, I review the role of fibroblasts in salamander limb regeneration and how their activity compares with that of human fibroblasts. In addition, the question of whether salamander blastema tissue could induce regeneration and tumor regression in animals with limited regeneration ability is discussed. A deeper understanding of these processes may lead to the development of novel regenerative and anticancer 
therapies.

\section{The Role of Fibroblasts in Salamander Limb Regeneration}

The regeneration of a complex body structure like a salamander limb can be divided into three different phases [6]. Following limb amputation, in the first phase epithelial cells migrate to cover the exposed underlying tissue, forming an epithelium that closes the wound. This wound epithelium then thickens, forming an apical epidermal cap (AEC). After wound closure, the regeneration process proceeds to the second phase, in which a population of undifferentiated cells migrates under the wound epidermis, leading to the formation of a structure called a blastema. Finally, in the third phase, the blastema grows in a proximodistal direction, regenerating the missing limb. Previous studies suggest that the most abundant cells in the blastema are connective tissue fibroblasts that have undergone dedifferentiation $[5,6]$. The other identified cell types seem to act as lineage-restricted tissue-specific stem cells [5]; however, it was reported that muscle satellite cells may act as a multipotent cell population [7]. Interestingly, muscle tissue appears to originate from both resident muscle satellite cells and dedifferentiated muscle cells [5]. Thus, along with dedifferentiation of mature cells, activation of reserve stem cell populations may also contribute to blastema formation.

The underlying reason for the significant presence of fibroblast-derived cells in the blastema remains unclear, however. It was suggested that these cells act as multipotent stem cells and are therefore involved in the regeneration of various tissues [6]. In this regard, fibroblast-derived blastema cells may play important structural roles. Limb regeneration in adult salamanders likely involves precise repetition of specific embryonic developmental steps to perfectly regenerate a limb. This precise process may be achieved by creating an embryonic-like environment at the site of regeneration. Therefore, it is possible that dedifferentiated fibroblasts in the blastema may also function to generate embryonic-like scaffolds, thus providing structural support for embryonic-like limb development. Interestingly, the information required to recreate the limb pattern is carried by dermal fibroblasts. This was shown by experiments in which regeneration was induced in irradiated limbs that received a graft of unirradiated skin [8]. Because irradiation inhibits cell proliferation, cellular contribution from the limb is blocked; thus, the grafted skin provides the only source of cells for the blastema. Surprisingly, this results in regeneration of a muscleless but otherwise normally patterned limb [8]. An important characteristic of blastema cells is positional identity, which ensures the correct pattern of tissue growth. A blastema only regenerates structures that are distal to the amputation level; accordingly, only the missing structures are regenerated [8]. Retinoic acid treatment can affect this positional identity by proximalizing blastema positional values [8]. For example, retinoic acid treatment of a blastema formed at the wrist level can result in a whole new limb regenerating from the wrist (with duplication of structures that are proximal to the amputation level). HOX genes seem to be involved in positional identity [8]. In fact, analysis of HOX expression in human fibroblasts suggests that these cells play an important role in defining skin positional identity [9]. Therefore, fibroblasts may constitute a positional identity map of the whole organism; accordingly, fibroblast-derived blastema cells may carry the positional information, thus directing blastema differentiation into appropriate mature tissues.

Dedifferentiated fibroblasts are the earliest blastema cells [5]; therefore, resident fibroblasts may be the earliest target of signals that initiate blastema formation. There are two main sources of signals that drive the formation of a blastema and then maintain its growth: the nerves and the AEC. Evidence suggests that fibroblast growth factors such as FGF-1, FGF-2, FGF-8, and FGF-10 are mediators of AEC signals [8], while FGF-2, glial growth factor 2 (GGF-2), substance $P$, tranferrin, and newt anterior gradient protein (nAG) act as mediators of nerve signals $[8,10]$. Interactions between the AEC and the severed limb nerves are essential for blastema formation. However, once formed and innervated, a blastema can produce its own neural factors; in fact, a blastema is an autonomous, self-organizing structure [8]. Interestingly, if a limb is denervated and then amputated, it is possible to rescue limb regeneration by artificially inducing nAG expression; however, the regenerated limb is usually atrophic [10]. As previously mentioned, regenerated structures are always formed in a proximodistal direction, and the most distal structure of a blastema is the AEC. This suggests that beneath the AEC there is a reservoir of proliferating blastema cells, which are maintained in an undifferentiated state by signals from the AEC. It is thought that during limb regeneration, these cells give rise to blastema cells that are at more proximal levels [5]; however, the blastema cells that grow in these more proximal regions are not under the influence of AEC signals and therefore differentiate into mature tissues [5]. In fact, evidence suggests that AEC signaling is spatially restricted [5]. The stem cell-like plasticity of fibroblast cells seems to be the key to the advanced regeneration ability of salamanders. In this regard, the limited regeneration ability of mammals may reflect an evolu- 
tionary loss of this fibroblast characteristic. Although human fibroblasts do not have the ability to dedifferentiate and initiate blastema formation, they are essential for promoting and supporting cancer stemness [11]. This shows that human fibroblasts retain the ability to support cell dedifferentiation processes.

\section{Salamander Regeneration and Cancer}

Salamander regeneration involves dedifferentiation of mature tissue into a mass of stem-cell like cells, which then redifferentiate into appropriate mature tissues to perfectly repair the damage. This precise process can be repeated indefinitely without resulting in abnormal tissue growth, whereas in mammals constant repair of tissue exposed to chronic damage has been linked to cancer [3]. Thus, many authors hypothesized that salamanders are resistant to cancer formation, leading to the use of salamanders as an in vivo animal model for cancer studies. These experiments involved transplantation of frog tumor tissue [12] as well as the use of cancer-inducing agents such as chemical carcinogens [3]. In one study [12], frog renal tumor tissue was transplanted subcutaneously into the salamander forelimb and began to grow and invade tissues. The limb was then amputated through the tumor mass, leading to regeneration of a normal limb and disappearance of the cancer. Histological studies indicated that frog tumor cells in the regenerated limb reverted to a normal phenotype and generated different tissues, such as muscle and cartilage [12]. This important result suggested that the salamander regeneration process can reverse tumorigenicity. Another study showed that in salamanders, chemically induced epithelial cancer can spontaneously revert to a normal phenotype [13], further supporting the idea that regeneration can reverse tumorigenicity. As previously mentioned, salamanders may be able to create an embryonic-like environment at the site of regeneration, thus allowing precise repetition of specific embryonic developmental steps to perfectly regenerate complex structures such as limbs. Considering that embryonic environments can reverse tumorigenicity by reprogramming tumor cells [14], the resistance to cancer of these animals may simply be a consequence of their regeneration ability rather than a specialized defense mechanism against cancer. In fact, administration of chemical carcinogens has been reported to induce cancer in regeneration-incompetent tissues but not in tissues capable of regeneration [3]. In particular, because the lens is regenerated from the dorsal but not the ventral iris, a few studies investigated tumor formation in these tissues after lens removal [3]. The results showed that administration of chemical carcinogens was able to induce formation of malignant tissue from the ventral but not the dorsal iris [3].

\section{Human Fibroblasts and Their Role in Wound Healing and Cancer}

Solid tumors have been described as "wounds that do not heal" [15]. In fact, wound healing and cancer share a number of common features, such as cell proliferation, angiogenesis, tissue remodeling, and a heavy involvement of fibroblasts. The physiological wound healing process can be divided into four different phases: hemostasis, inflammation, proliferation, and tissue remodeling [16]. The main function of the hemostasis phase is to stop bleeding, which is accomplished through the process of coagulation. Following wounding, the first response of damaged blood vessels is vasoconstriction, which is induced by different mediators, such as endothelin (produced by the vascular endothelium) and noradrenaline (released by sympathetic nerves) [17]. Next, blood contact with the exposed endothelial collagen triggers the coagulation process; platelets adhere to the damaged surface to form a plug that seals the vessel, and a fibrin matrix is deposited to further strengthen the platelet plug $[17,18]$. The fibrin clot is not only important to stop blood loss, but also to provide a provisional matrix for granulation tissue formation and wound re-epithelization $[17,18]$. In addition, activated platelets release growth factors and cytokines for the activation and recruitment of leukocytes, endothelial cells, and fibroblasts [18]. The main function of the inflammation phase is to clean and sterilize the wound; pathogens, cellular debris, and foreign materials are removed by phagocytic cells such as neutrophils and macrophages [17]. During this phase, mediators such as kinins, histamine, prostaglandins, and leukotrienes stimulate vasodilatation to facilitate the extravasation of circulating cells [17]. Neutrophils are mainly present during the inflammation phase and are then removed by apoptosis, while macrophages remain until wound healing is complete [17]. Macrophages play an essential role during the successive phases of wound healing, especially in supporting fibroblast activity though the release of important molecules such as transforming growth factor-beta (TGF-beta) [19]. Once the inflammatory response begins to subside, the proliferation phase of healing begins. Resolution of the inflammatory response is essential for successful healing [20]. The main function of the proliferation phase is to repair the damaged tissue; this starts with the formation of granulation tissue, which consists essentially of infiltrating cells (e.g., fibroblasts and macrophages), proliferating blood vessels, and loose connective tissue [21]. As previously mentioned the fibrin clot provides a provisional matrix for granulation tissue formation 
and wound re-epithelization. In particular, the fibrin clot is rich in fibronectin, which appears to be an important protein that supports the infiltration of fibroblasts and keratinocytes [22, 23]. During the proliferation phase, fibroblasts infiltrate the wound and release matrix metalloproteinases (MMPs) to degrade the fibrin clot, allowing deposition of newly synthesized extracellular matrix [21]; this initial matrix is weaker than that of later stages, probably to facilitate angiogenesis and cell invasion. Specifically, the early matrix is rich in fibronectin and hyaluronic acid, while the later matrix is rich in collagens and proteoglycans [21]. Under the influence of factors such as TGF-beta, a portion of fibroblasts then acquire the myofibroblast phenotype, which is characterized by the expression of alpha-smooth muscle actin (alpha-SMA) [24]. Myofibroblasts greatly contribute to the synthesis of new extracellular matrix [25]. In addition, these cells generate contractile force to narrow the gap between the wound edges [25]. In vitro studies indicate that in fibroblasts, alpha-SMA expression enhances the generation of contractile force [26]. Increased contractile force appears to be a hallmark of scar formation. In fact, scarless wound healing in salamanders is characterized by the presence of a very small number of myofibroblasts in the wound [27], indicating a reduced need for wound contraction; by contrast, when macrophages are artificially depleted after wounding, which hampers salamander limb regeneration and leads to scar formation, an increased number of myofibroblasts is observed in the wound [28]. Interestingly, scarless wound healing in mammalian fetuses is also characterized by an evident lack of myofibroblasts $[29,30]$. At the end of the proliferation phase, myofibroblasts undergo apoptosis and are cleared by macrophages, leading to scar formation [31]. The final phase of wound healing is tissue remodeling, which aims to maximize restoring of the pre-existing tissue [17]. In this phase, fibroblasts continue to remodel the extracellular matrix, and the duration of this process depends on the type of wound [18]. The healed tissue can usually regain approximately $80 \%$ of its original strength $[17,18]$.

In contrast with physiological wound healing, a process at the end of which myofibroblasts undergo apoptosis and are cleared from the healed tissue, in cancer the myofibroblasts present in the tumor stroma are maintained in a state of persistent activation by the tumor [32]. These cells in the tumor stroma are called cancer-associated fibroblasts (CAFs) [32]. Activated CAFs support tumor growth by secreting important molecules such as growth factors, cytokines, and proteases; the reciprocal interplay between CAFs and tumor cells forms the basis for tumor progression and metastasis. Among the tu- mor-supporting molecules produced by CAFs, TGF-beta, stromal cell-derived factor-1 (SDF-1), hepatocyte growth factor (HGF), vascular endothelial growth factor (VEGF), fibroblast growth factor (FGF), and proteases such as MMPs play a crucial role in tumorigenesis [32]. TGF-beta appears to increase the levels of CXCR4 expression in tumor cells, thus enhancing tumor cell sensitivity to SDF-1, a growth factor that specifically binds to the CXCR4 receptor [33]. However, TGF-beta has also been reported to inhibit tumor growth, while at the same time promoting metastasis [34]. HGF promotes invasion by binding to c-MET, a tyrosine kinase receptor typically expressed by epithelial-derived tumors such as esophageal squamous cell carcinoma [35]. VEGF promotes angiogenesis, an essential process that provides oxygen and nutrients to the tumor [36]. FGF has been shown to be associated with cancer progression in several experimental models. For example, binding of FGF-2 to its receptor activates the progesterone receptor in mouse mammary tumor cells, thus promoting tumor proliferation [37]; in addition, FGF-2 stimulates proliferation of human breast cancer cells as well [37]. MMPs cause proteolytic degradation of the extracellular matrix, allowing the tumor to grow and metastasize [38]. Furthermore, cleavage of the extracellular matrix by MMPs can cause additional release of tumor-supporting molecules such as VEGF [32]. In addition to providing tumor-supporting molecules, CAFs are thought to actively participate in the tumor metabolism. Specifically, evidence suggests that CAFs are induced by the tumor to undergo aerobic glycolysis, thus providing energy-rich metabolites such as lactate and pyruvate, which are then used by cancer cells in the Krebs cycle [32].

\section{Conclusions and Future Directions}

Salamanders are a valuable animal model to study phenomena such as cancer and regeneration. The ability of salamanders to regenerate tissue provides a model for regenerative medicine. The ability of salamanders to reverse tumorigenicity can help us understand how to manipulate the biological conditions that cause and maintain cancer. Considering that signaling pathways involved in regeneration may be highly conserved among all vertebrates, an interesting question is whether salamander blastema tissue could induce regeneration and tumor regression in animals with limited generation ability. However, considering mammals as potential hosts, there are a number of possible limitations. A first issue is that host body temperature will probably dictate whether salamander blastema cells can successfully grow in the host. However, it may be possible to engineer blastema cells to grow at different temperatures, while initial 
studies could be conducted using ectothermic animals such as frogs as hosts. A second issue is the host immune system reaction against the transplanted tissue. However, this problem may be addressed by using immunosuppressive agents. Another vital question is whether host tissues can support the regeneration process of a salamander blastema. Interestingly, blastema autografting and homografting experiments show that, once developed, a blastema can act as an autonomous, self-organizing structure [8]; thus, a xenotransplanted blastema may successfully grow, provided that it can rely on the host tissues for nutrients. Furthermore, addition of the nAG protein may facilitate the process. However, using an already developed blastema poses the additional problem of whether the regenerated limb will be a salamander limb, a normal limb, or a chimeric limb. This is a crucial aspect because the host immune system may reject the new limb. In this regard, an alternative could be to induce blastema formation directly in the host. Considering their essential role in blastema formation, salamander fibroblasts may be able to initiate blastema formation at the site of the host tissue wound, resulting in a blastema composed mainly of undifferentiated host cells. Furthermore, bone marrow-derived cells could act as a source of fibroblasts for wounds and tumors [39]. Therefore, it may also be worthwhile to investigate xenotransplantation of engineered salamander bone marrow stem cells as a therapeutic strategy for inducing a salamander-like regenerative and anticancer response.

\section{Acknowledgments}

This work was supported by Innovative Bioresearch, Milan, Italy.

\section{Competing Interests}

The author has declared that no competing interest exists.

\section{References}

1. Lee K, Nelson CM. New insights into the regulation of epithelial-mesenchymal transition and tissue fibrosis. Int Rev Cell Mol Biol. 2012; 294 : 171-221.

2. Roy S, Lévesque M. Limb regeneration in axolotl: is it superhealing? Sci World J. 2006; 6(Suppl 1): 12-25.

3. Oviedo NJ, Beane WS. Regeneration: the origin of cancer or a possible cure? Semin Cell Dev Biol. 2009; 20: 557-64.

4. Barile L, Messina E, Giacomello A, Marbán E. Endogenous cardiac stem cells. Prog Cardiovasc Dis. 2007; 50: 31-48.

5. McCusker C, Gardiner DM. The axolotl model for regeneration and aging research: a mini-review. Gerontology. 2011; 57: 565-71.

6. Bryant SV, Endo T, Gardiner DM. Vertebrate limb regeneration and the origin of limb stem cells. Int J Dev Biol. 2002; 46: 887-96.

7. Morrison JI, Borg P, Simon A. Plasticity and recovery of skeletal muscle satellite cells during limb regeneration. FASEB J. 2010; 24: 750-6.

8. Nye HL, Cameron JA, Chernoff EA, Stocum DL. Regeneration of the urodele limb: a review. Dev Dyn. 2003; 226: 280-94.

9. Rinn JL, Wang JK, Allen N, Brugmann SA, Mikels AJ, Liu H, Ridky TW, Stadler HS, Nusse R, Helms JA, Chang HY. A dermal HOX transcriptional program regulates site-specific epidermal fate. Genes Dev. 2008; 22: 303-7.
10. Kumar A, Godwin JW, Gates PB, Garza-Garcia AA, Brockes JP. Molecular basis for the nerve dependence of limb regeneration in an adult vertebrate. Science. 2007; 318: 772-7.

11. Giannoni E, Bianchini F, Masieri L, Serni S, Torre E, Calorini L, Chiarugi P. Reciprocal activation of prostate cancer cells and cancer-associated fibroblasts stimulates epithelial-mesenchymal transition and cancer stemness. Cancer Res. 2010; 70: 6945-56.

12. Rose SM, Wallingford HM. Transformation of renal tumors of frogs to normal tissues in regenerating limbs of salamanders. Science. 1948; 107: 457.

13. Seilern-Aspang F, Kratochwil K. Induction and differentiation of an epithelial tumour in the newt (Triturus cristatus). J Embryol Exp Morphol. 1962; 10: 337-56.

14. Hendrix MJ, Seftor EA, Seftor RE, Kasemeier-Kulesa J, Kulesa PM, Postovit LM. Reprogramming metastatic tumour cells with embryonic microenvironments. Nat Rev Cancer. 2007; 7: 246-55.

15. Dvorak HF. Tumors: wounds that do not heal. Similarities between tumor stroma generation and wound healing. N Engl J Med. 1986; 315: 1650-9.

16. Guo S, DiPietro LA. Factors affecting wound healing. J Dent Res. 2010; 89: 219-29.

17. Teller P, White TK. The physiology of wound healing: injury through maturation. Surg Clin North Am. 2009; 89: 599-610.

18. Velnar T, Bailey T, Smrkolj V. The wound healing process: an overview of the cellular and molecular mechanisms. J Int Med Res. 2009; 37: 1528-42.

19. Rodero MP, Khosrotehrani K. Skin wound healing modulation by macrophages. Int J Clin Exp Pathol. 2010; 3: 643-53.

20. Eming SA, Krieg T, Davidson JM. Inflammation in wound repair: molecular and cellular mechanisms. J Invest Dermatol. 2007; 127: 514-25.

21. Clark RAF. Wound repair: overview and general considerations. In: Clark RAF, ed. The molecular and cellular biology of wound repair, 2nd ed. New York: Plenum Press; 1996: 3-50.

22. Greiling D, Clark RAF. Fibronectin provides a conduit for fibroblast transmigration from collagenous stroma into fibrin clot provisional matrix. J Cell Sci. 1997; 110: 861-70.

23. Grinnell F, Toda K, Takashima A. Activation of keratinocyte fibronectin receptor function during cutaneous wound healing. J Cell Sci. 1987; 8(Suppl): 199-209.

24. Martin P. Wound healing-aiming for perfect skin regeneration. Science. 1997; 276: 75-81.

25. Li B, Wang JH. Fibroblasts and myofibroblasts in wound healing: force generation and measurement. J Tissue Viability. 2011; 20: 108-20.

26. Hinz B, Celetta G, Tomasek JJ, Gabbiani G, Chaponnier C. Alpha-smooth muscle actin expression upregulates fibroblast contractile activity. Mol Biol Cell. 2001; 12: 2730-41.

27. Seifert AW, Monaghan JR, Voss SR, Maden M. Skin regeneration in adult axolotls: a blueprint for scar-free healing in vertebrates. PLoS One. 2012; 7: e32875.

28. Godwin JW, Pinto AR, Rosenthal NA. Macrophages are required for adult salamander limb regeneration. Proc Natl Acad Sci USA. 2013; 110: 9415-20.

29. McCluskey J, Martin P. Analysis of the tissue movements of embryonic wound healing-DiI studies in the limb bud stage mouse embryo. Dev Biol. 1995; 170: 102-14.

30. Cass DL, Sylvester KG, Yang EY, Crombleholme TM, Adzick NS. Myofibroblast persistence in fetal sheep wounds is associated with scar formation. J Pediatr Surg. 1997; 32: 1017-22.

31. Desmoulière A, Redard M, Darby I, Gabbiani G. Apoptosis mediates the decrease in cellularity during the transition between granulation tissue and scar. Am J Pathol. 1995; 146: 56-66.

32. Cirri P, Chiarugi P. Cancer associated fibroblasts: the dark side of the coin. Am J Cancer Res. 2011; 1: 482-97.

33. Ao M, Franco OE, Park D, Raman D, Williams K, Hayward SW. Cross-talk between paracrine-acting cytokine and chemokine pathways promotes malignancy in benign human prostatic epithelium. Cancer Res. 2007; 67: 4244-53.

34. Stuelten $\mathrm{CH}$, DaCosta Byfield S, Arany PR, Karpova TS, Stetler-Stevenson WG, Roberts AB. Breast cancer cells induce stromal fibroblasts to express MMP-9 via secretion of TNF-alpha and TGF-beta. J Cell Sci. 2005; 118: 2143-53.

35. Grugan KD, Miller CG, Yao Y, Michaylira CZ, Ohashi S, Klein-Szanto AJ, Diehl JA, Herlyn M, Han M, Nakagawa H, Rustgi AK. Fibroblast-secreted hepatocyte growth factor plays a functional role in esophageal squamous cell carcinoma invasion. Proc Natl Acad Sci USA. 2010; 107: 11026-31.

36. Dong J, Grunstein J, Tejada M, Peale F, Frantz G, Liang WC, Bai W, Yu L, Kowalski J, Liang X, Fuh G, Gerber HP, Ferrara N. VEGF-null cells require PDGFR alpha signaling-mediated stromal fibroblast recruitment for tumorigenesis. EMBO J. 2004; 23: 2800-10.

37. Giulianelli S, Cerliani JP, Lamb CA, Fabris VT, Bottino MC, Gorostiaga MA, Novaro V, Góngora A, Baldi A, Molinolo A, Lanari C. Carcinoma-associated fibroblasts activate progesterone receptors and induce hormone independent mammary tumor growth: a role for the FGF-2/FGFR-2 axis. Int J Cancer. 2008; 123: 2518-31.

38. Katiyar SK. Matrix metalloproteinases in cancer metastasis: molecular targets for prostate cancer prevention by green tea polyphenols and grape seed proanthocyanidins. Endocr Metab Immune Disord Drug Targets. 2006; 6: 17-24.

39. Ishii G, Sangai T, Sugiyama K, Ito T, Hasebe T, Endoh Y, Magae J, Ochiai A. In vivo characterization of bone marrow-derived fibroblasts recruited into fibrotic lesions. Stem Cells. 2005; 23: 699-706. 